\title{
Art public et mémoire collective
}

Warburg et le Monument à Bismarck de Hambourg (1906)

\section{Micol Forti}

\section{CpenEdition}

\section{Journals}

Édition électronique

URL : http://journals.openedition.org/imagesrevues/3004

DOI : 10.4000/imagesrevues.3004

ISSN : $1778-3801$

Éditeur :

Centre d'Histoire et Théorie des Arts, Groupe d'Anthropologie Historique de l'Occident Médiéval, Laboratoire d'Anthropologie Sociale, UMR 8210 Anthropologie et Histoire des Mondes Antiques

\section{Référence électronique}

Micol Forti, «Art public et mémoire collective », Images Re-vues [En ligne], Hors-série 4 | 2013,

document 1, mis en ligne le 30 janvier 2013, consulté le 30 janvier 2021. URL : http://

journals.openedition.org/imagesrevues/3004; DOI : https://doi.org/10.4000/imagesrevues.3004

Ce document a été généré automatiquement le 30 janvier 2021.

\section{(c) (7) (8)}

Images Re-vues est mise à disposition selon les termes de la Licence Creative Commons Attribution Pas d'Utilisation Commerciale 4.0 International. 


\title{
Art public et mémoire collective
}

\author{
Warburg et le Monument à Bismarck de Hambourg (1906)
}

\section{Micol Forti}

Le 29 janvier 1902, Warburg publie, dans le Hamburgische Correspondenten, un article intitulé «La nuit de Valpurga sur le Stintfang ${ }^{1}$, signé de la courte abréviation "W.Fl." (à savoir "Warburg-Florence"). Le texte fait référence à la présentation effectuée le 5 janvier, au vélodrome proche de la gare de Dammtor, de 239 projets pour la réalisation à Hambourg d'un monument à Bismarck. Le concours est remporté par le sculpteur Hugo Lederer et l'architecte Emil Schaudt qui réaliseront le monument inauguré en juin 1906 (Fig.1-2).

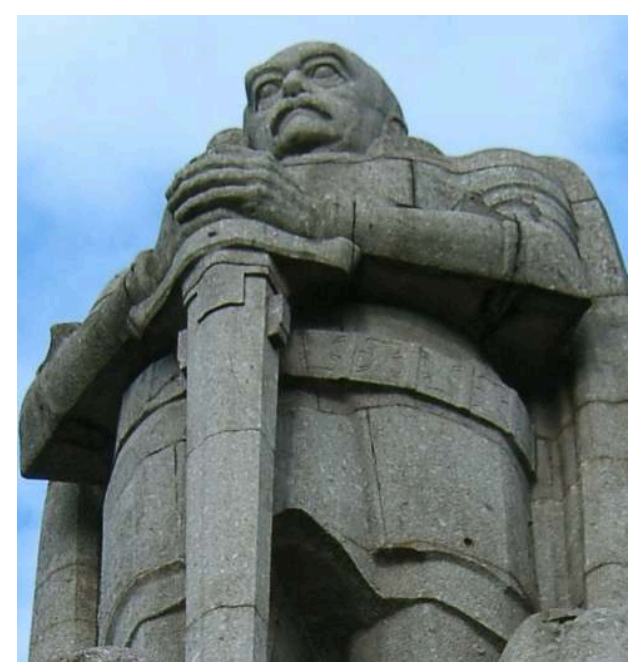


Fig.1

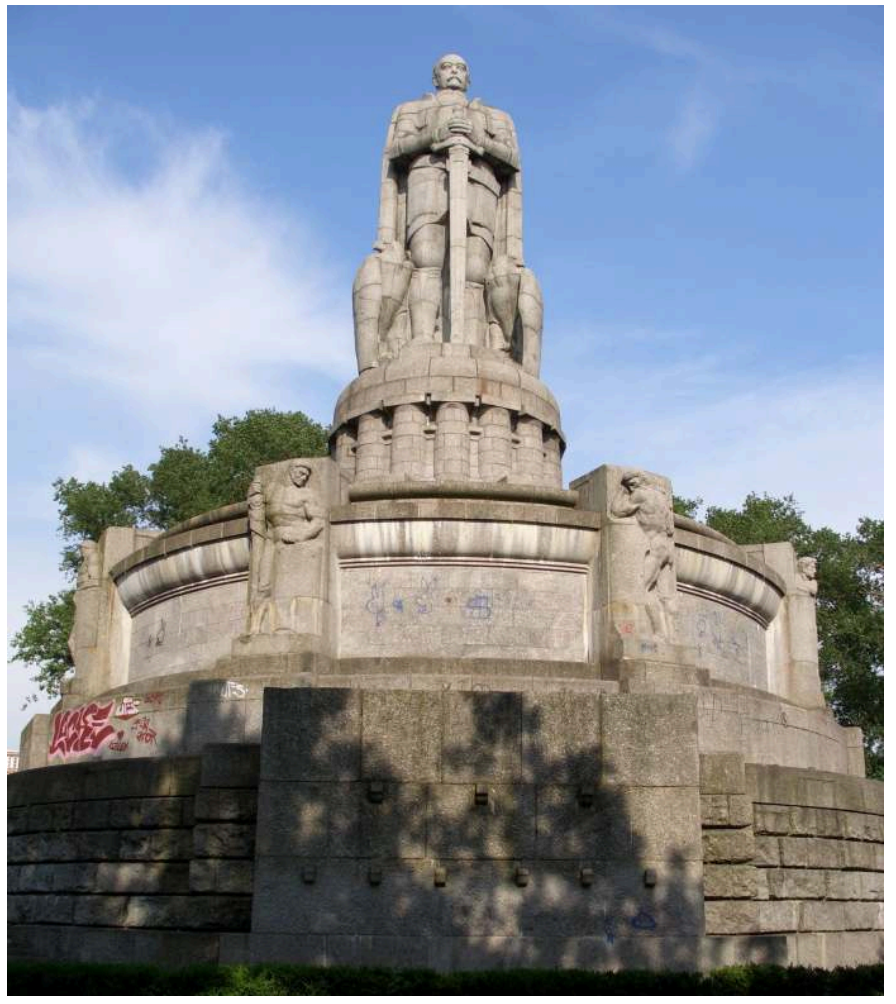

Hugo Lederer et Emil Schaudt, Monument à Otto von Bismarck, 1902-1906, Amburgo

Fig.2

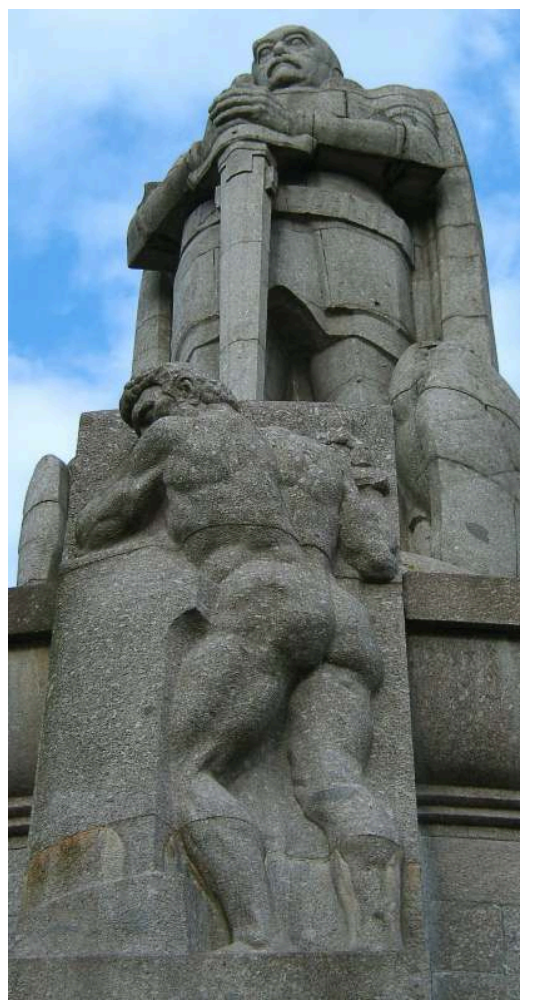

Hugo Lederer et Emil Schaudt, Monument à Otto von Bismarck, 1902-1906, Amburgo, detail. 
2 L'article n'est pas une simple chronique. Warburg souhaite prendre part au débat qui s'ensuit et répondre aux nombreuses critiques et invectives consécutives au choix du jury qui est aussi le sien. Mais, au même temps, il veut réfléchir autour des enjeux théoriques posés par la réalisation d'un monument public qui célèbre un personnage central de l'histoire contemporaine allemande.

3 Conformément à ses habitudes, vérifiées à de nombreuses reprises à propos de l'art contemporain et de la vie culturelle de sa propre ville, son intérêt pour cette grande entreprise publique se manifeste dès les phases préliminaires politico-administratives choix du lieu où ériger le monument, sélection des membres du jury - pour se porter ensuite sur les différentes étapes d'exécution. Il échange des avis avec de très nombreux correspondants épistolaires, accumulant un gigantesque fonds composé d'articles, photographies, coupures de presse en tout genre, qui constitueront un Album grâce auquel il est aujourd'hui possible de parcourir à nouveau les différentes phases du débat relatif au monument de Hambourg ${ }^{2}$. Il va jusqu'à consacrer un secteur entier de sa bibliothèque aux ouvrages historiques, politiques ou économiques relatifs au Chancelier - tels les livres d'Erich Marcks, convié par Warburg en 1929 à donner une conférence sur "Bismarck et l'antiquité classique"3 - mais également à ses biographies, psychologico-caractérielles et "mythographiques", incluant un superbe ouvrage français de caricatures de Bismarck classées par pays européens et États-Unis ${ }^{4}$.

4 La bibliographie, établie d'après la thèse de doctorat, publiée par la suite, de Mark Russell ${ }^{5}$, jusqu'aux travaux détaillés de Jörg Schilling ${ }^{6}$ et Claudia Wedepohl ${ }^{7}$, retrace, selon différents points de vue, les étapes du concours et du monument, indissociables du phénomène général de la profusion de sculptures commémoratives dédiées à Bismarck dans toute l'Allemagne et ses colonies.

5 A partir de ça, ce que je voudrais proposer, c'est une analyse tant des textes que du matériel récoltés par Warburg à ce sujet, qui puisse donner de nouveaux éléments d'interprétation des moyens et des buts propres à l'historien d'art spécialiste de la Renaissance quand il s'est consacré aux événements culturels de son époque.

6 Jörg Schilling, tout comme Claudia Wedepohl, n'ont certainement pas tort quand ils affirment que, dans l'ensemble, le jugement critique de Warburg sur le monument dédié à Bismarck est plutôt vague, voire franchement ambigu par ses aspects polémiques, et que sa contribution théorique sur les éléments formels, que nous analyserons par la suite, n'offre qu'une vision indécise rendant difficile toute évaluation de l'objet d'art. On retiendra toutefois que certaines thématiques mises en évidence par Warburg, même si elles ne furent pas explicitées par un raisonnement construit, s'avérèrent déterminantes pour clarifier l'importance historico-culturelle et les implications théoriques liées à cet événement.

7 Warburg amorce son article avec une critique féroce et irrespectueuse à l'encontre des voix « de l'homme simple plein de bons sentiments et de sa famille » qui contestèrent le choix du jury de récompenser le projet de Lederer et Schaudt. Ses propos vont au-delà de son aversion, manifestée en d'autres circonstances, pour le goût petit bourgeois de la classe moyenne de Hambourg, ce qui pose un problème essentiel, c'est à dire celui du rapport spécial qui s'instaure entre une œuvre d'art officielle, installée dans un espace extérieur, et son public.

8 Le public a des attentes qui correspondent à des critères particuliers : des critères de vérité, comme les définira Gombrich ${ }^{8}$. Ils déterminent et sont déterminés par des 
"attentes" ponctuelles de la part du bénéficiaire : «Ceci est-il mon Bismarck ? Ceci estil notre Bismarck ? [...] Nous voulons voir dans ce monument ce que Bismarck a fait et éprouvé », écrit Warburg, singeant les propos de la foule incarnée par le «brigadier adjoint aux lourdes bottes ».

9 De telles attentes évaluent l'œuvre au regard de sa capacité d'évocation, de mémoire, de ce qu'elle représente. Le public réclame un « effet de présence » : représenter, « c'est au fond opérer une substitution, la substitution de quelque chose à la place de cette autre [...] faire comme si $»^{9}$, pour citer les propos avec lesquels Louis Marin définit l'un des sens du mot représenter.

Warburg semble souligner une séparation de la grande tradition illuministe, celle qui conduit Louis de Jaucourt à écrire dans l'Encyclopédie, on appelle monument «tout ouvrage d'architecture et de sculpture fait pour conserver la mémoire des hommes illustres ou des grands événements, comme un mausolée, une pyramide, un arc-detriomphe et autres semblables $»^{10}$. Le monument, c'est d'abord et fondamentalement un lieu de mémoire.

11 Une séparation déterminée par le changement du rôle de la population hambourgeoise des premières années $\mathrm{du} \mathrm{XX}^{\mathrm{e}}$ siècle - celle que Baudelaire définit comme "foule" qui s'organise en tant que public et accède au rôle de commanditaire.

12 La question de l'identité entre image et objet, induite dans le principe, plus global et épineux, de la "ressemblance", est invoquée à grands cris par les citoyens de Hambourg à travers l'exaltation de réels principes "extérieurs", comme l'illustrent la très grande majorité des monuments dans les différentes villes dédiés au Chancelier. Cette demande sous-tend une signification majeure : la possibilité/nécessité de "reconnaître" est considérée comme un préalable indispensable pour "se souvenir". De ce fait, la "mémoire collective" devient le résultat d'exigences subjectives et de pouvoirs objectifs attribués à la vision.

13 Parmi les aspects les plus appréciés dans le personnage de Bismarck du monument réalisé par Reinhold Begas à Berlin (Fig.3), hormis son portrait et la savante composition allégorique, se trouvent surtout deux gestes, deux attitudes grâce auxquels les citoyens "reconnaissent", car ils l'ont "connu", leur héros : le mouvement de la nuque tournée d'un côté, et les doigts de la main légèrement écartés sur la garde de l'épée ${ }^{11}$. 
Fig.3

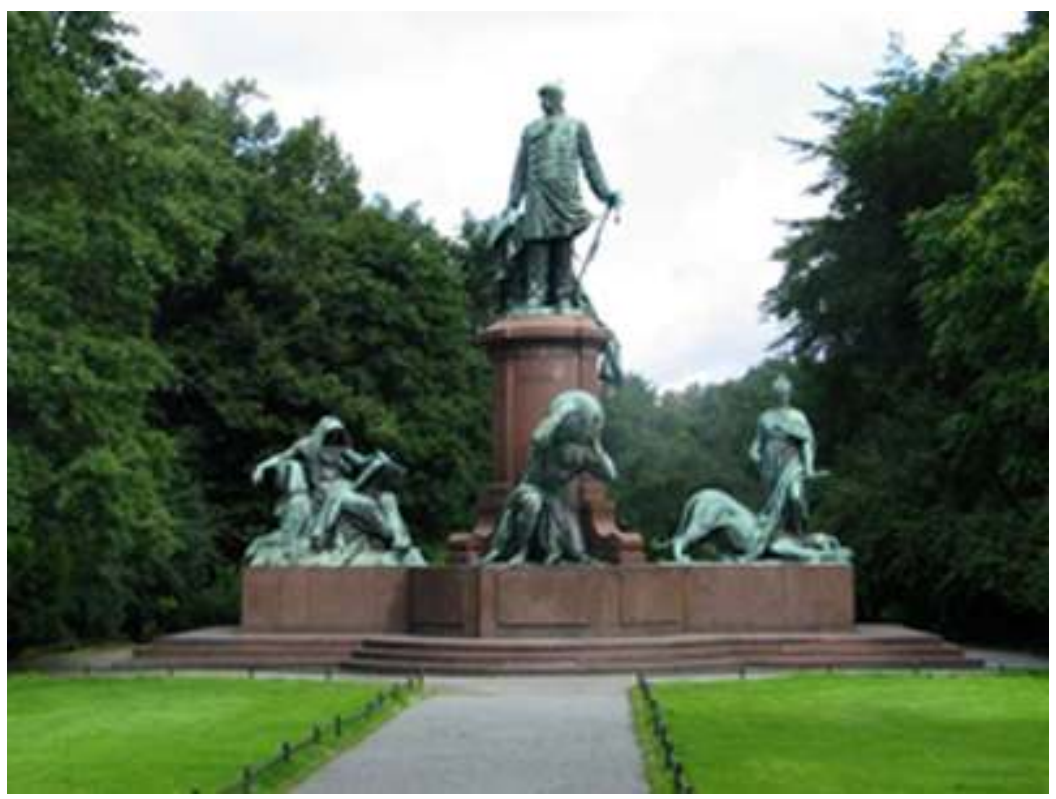

Reinhold Begas, Monument à Otto von Bismarck, 1901, Berlin

Et c'est précisément sur ce point que Warburg tente d'intervenir, en proposant un point de vue qui pousse le public, auquel le monument doit répondre et avec lequel il doit interagir, à considérer d'autres voies d'interprétation, d'autres modalités pour traduire les méandres de la mémoire collective, dans le cas particulier de l'édification d'un monument devant représenter la ville, la grandeur de la nation, la force de l'État. Dans son essai L'art du portrait et la bourgeoisie florentine, du 1902, Warburg affirme que les œuvres d'art sont le résultat de la collaboration entre «les commanditaires et les artistes » : c'est à dire qu'elles sont dès l'origine le fruit d'un compromis ${ }^{12}$.

Il convient de souligner que Warburg insiste à différentes reprises ${ }^{13}$ sur le fait que le Monument à Bismarck puisse représenter la «renaissance », le "virage critique », la "formation d'un goût indépendant " dans le développement de "l'art monumental allemand». Il s'agit d'une allégation qui implique la conjugaison de différents concepts : celui de la renaissance, celui de la renaissance d'un art identifié comme allemand, celui d'une renaissance associé à une œuvre sculptée et tout particulièrement à un monument commémoratif.

Nous partirons de ce dernier point.

17 La réflexion théorique autour de la sculpture avait permis, aux XVIII e et XIX ${ }^{e}$ siècles, d'intéressantes percées quant à la définition d'un langage autonome, mais était alors encore profondément ancré un jugement fondamentalement sceptique et négatif sur la possibilité de la sculpture à devenir un art en position de répondre aux exigences de la modernité. Les critiques de Zola $^{14}$ et Huysmans ${ }^{15}$, conjuguées à celles, bien plus radicales et qui en constituent la genèse, de Baudelaire (au Salon de 1846, il écrit son célèbre essai Pourquoi la sculpture est ennuyeuse ${ }^{16}$ ) ou de Hegel dans son Esthétique, vont au-delà d'un jugement négatif limité aux œuvres, et remettent également en question la capacité expressive et le potentiel d'innovation de la sculpture en tant que telle et en rapport à une " esthétique de la modernité" par laquelle la sculpture ne semble pas être touchée pour avoir trouvé son acmé dans le passé, dans l'Antiquité classique, comme l'avait d'ailleurs démontré Winckelmann ${ }^{17}$. 

et partagé. Le naturalisme vide, qui « représente vraiment le niveau culturel le plus bas de l'activité artistique » et les principes expressifs basés sur le "théâtre de variétés et [la] pantomime de cirque ", sont des mécanismes de pur mimétisme, liés au simple souvenir et non à de plus généraux mais durables mécanismes de transmission d'une "mémoire": «Le Bismarck connu [...] est seulement une enveloppe temporelle, qui rend 
superficiellement familière » l'essence de sa présence et de son rôle vis-à-vis de la collectivité.

Ce sont ces mêmes dangers que Julius von Schlosser met en évidence dans son bel essai de 1926 sur le Culte moderne des monuments ${ }^{23}$. Il les avait déjà exprimés en 1911 dans son étude sur les portraits de $\operatorname{cire}^{24}$ : si on se limite à parler - écrit l'historien d'art autrichien - de " poses et gestes stéréotypés ", de "symboles dépouillés et accessoires de théâtre ", alors on reste "empêtré » dans un certain "extérieur » qui ne correspondra jamais avec le personnage représenté.

Il n'existe ni correspondance ni identité entre le détail réaliste, physionomique, anecdotique, et la capacité de l'œuvre d'art à transmettre, au-delà de son époque, la représentation d'un personnage, d'une idée, d'un événement. Ce n'est qu'en construisant une force d'expression et un vocabulaire dégagé des éléments qui font partie du récit et non de l'histoire, du moment et non du concept, du particulier et non de l'universel, que l'œuvre d'art pourra continuer à être représentative en-dehors de son propre contexte temporel.

Mais Warburg pressent d'autres dangers sous-jacents à ce qu'il définit comme la "mentalité mercantile petit bourgeois », soit la possibilité d'une corruption de l'image qui la réduise à un produit de consommation, de peu de valeur, fondu « dans le chocolat et dans le savon ${ }^{25}$ (Fig.4).

Fig.4

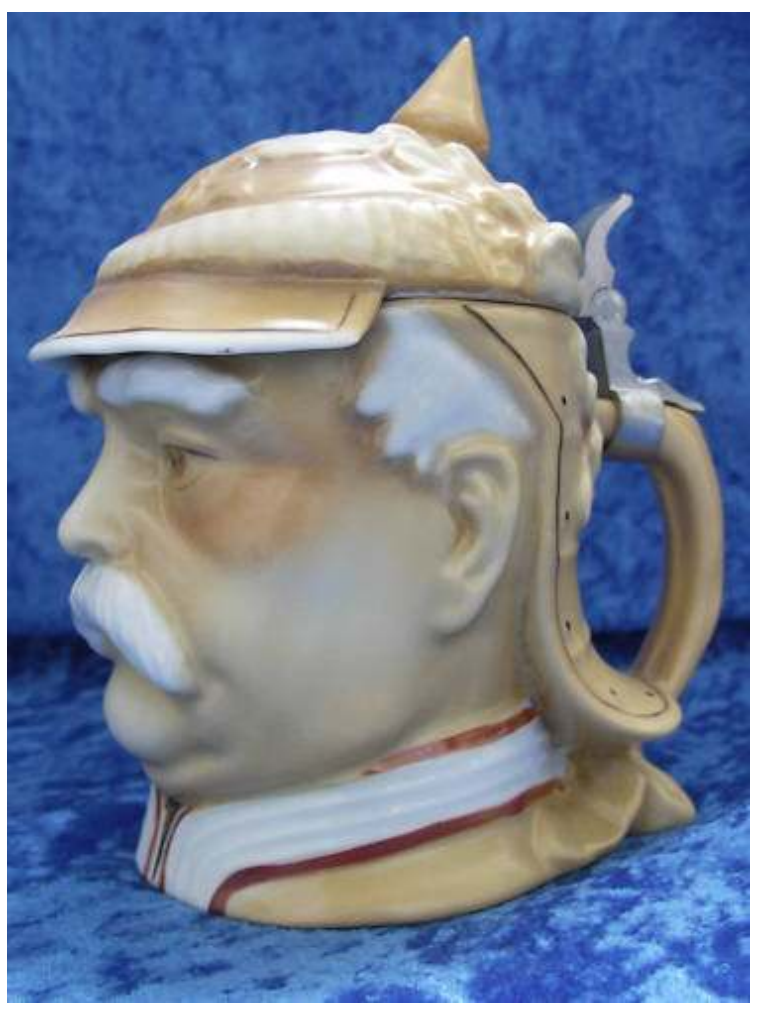

Cruchon en céramique qui représente le visage de Bismarck

29 Cette décadence semblerait aller de pair avec la domination aveugle des images établies sur une vague répétition "du style baroque », sur la modestie des formules allégoriques et sur l'évidence typologique des portraits « de photos instantanées ». C'est une réelle prévision de cette perte de « l'aura » de l'œuvre d'art et du risque d'une « esthétisation 
" de l'image du pouvoir, mais également de "démocratisation" de l'art, que Walter Benjamin ${ }^{26}$ formulera quelques décennies plus tard dans ses écrits, lui qui aurait tellement désiré participer aux recherches du Warburg Institute.

Si l'œuvre est le fruit d'un assujettissement aux requêtes du public - entendu dans son nouveau rôle de commanditaire mais également dans son immensité numérique et indifférenciée -, elle perdra son pouvoir de communiquer, exprimer, évoquer, voire intimider, du fait d'un système stratifié de renvois internes et des multiples possibilités de lecture qui constituent l'essence de son unicité et de son appartenance à l'histoire des images.

Elle sera l'expression d'une mémoire individuelle ou générationnelle, et non collective et culturelle, pour reprendre la distinction établie par Aleida Assmann dans son ouvrage Erinnerungsräume. Formen und Wandlungen des Kulturellen Gedächtnisses de $1999^{27}$.

Face à une œuvre contemporaine, Warburg est contraint à renverser le point de vue utilisé dans les travaux de l'époque, comme Échanges artistiques entre nord et sud au XVe siècle ${ }^{28}$, de 1905, ou postérieurs, comme dans les panneaux de Mnémosyne, pour lesquels même l'objet de consommation, l'image publicitaire, la photographie d'un journal, apparemment dégagés d'une référence directe à la culture dans l'acception la plus élitiste du mot, montrent de fait un lien indissociable avec l'histoire en tant « qu'espace de la mémoire ».

Le danger potentiel sur lequel le chercheur insiste ici est inhérent à la création d'un monument public commandé par une institution, jugé par une commission, destiné aux citoyens et, d'une façon plus générale, offert au public. La représentation de l'image de Bismarck est évaluée par rapport à l'objectif de transmission de principes et valeurs collectifs et constitutifs de la nation. L'identité expressive et formelle du monument et de l'image représentée soulève des questions esthétiques qui, prises dans l'engrenage d'une "formule esthétisante" basée sur la "ressemblance extérieure", la feraient disparaître dans le vague cadre d'un vocabulaire vériste/naturaliste qui cherche sa légitimité dans la reprise d'esthétiques néobaroques. La perte d'une conscience collective du rôle de l'œuvre publique constitue le premier pas vers une séparation de l'histoire des images et des idées, vers l'inexorable perte de "l'aura", vers la reproduction en série d'images commerciales désormais détachées de toute nécessité de transmission historico-culturelle.

Cette analyse critique est complétée par la pars construens qui voit Warburg participer, une fois de plus, au débat autour de questions relatives au vocabulaire contemporain, en particulier celui de la sculpture.

En fait - comme l'attestent de nombreux articles et le discours de Johann Georg Mönckeberg, maire de Hambourg et président de la commission d'évaluation, à l'occasion de l'attribution du prix en date du 10 janvier $1902-$, il ne se limite pas à remplacer l'image-portrait par la représentation d'un "personnage idéal", les détails physionomiques de l'image historique par des références à Roland et à la mythologie, ou à affirmer l'idée d'une essence germanique exprimée sous forme germanique ${ }^{29}$. Au contraire, Warburg propose d'évaluer la nécessité même d'une image, en particulier celle de Bismarck, à partir du fonctionnement général de l'œuvre à l'intérieur d'un système de perception : d'un " approfondissement de l'objet tenu à distance fixe ».

On a largement traité des relations que Warburg entretient avec Adolf von Hildebrand pendant les années de la période florentine à partir de 1897, tout comme de sa 
connaissance de l'ouvrage Le problème de la forme dans les arts plastiques ${ }^{30}$ de 1893 . On ne s'étonnera donc pas de la citation explicite d'Hildebrand au cours de l'article et du rappel de certains concepts fondamentaux de sa théorie visibiliste.

L'ouvrage d'Hildebrand s'inscrit dans une ligne théorique qui tend à reconsidérer l'art plastic-sculptural d'une manière autonome et non plus subordonnée à la peinture. Depuis la Lettre sur la sculpture du philosophe hollandais Franz Hermsterhuis, publiée en français en 1769, ou de la plus connue Plastique de Johann Gottfried Herder de 1778, en passant par les réflexions d'August Wilhelm Schlegel dans sa Doctrine de l'art de 1801, jusqu'à la Philosophie de l'art de Schelling de 1802-1804, puis aux Leçons d'esthétique de Hegel de 1818-1821, la production critique et philosophique avait déjà introduit quelques concepts fondamentaux parmi lesquels celui de la tactilité comme vecteur sensible, alternatif, parallèle ou complémentaire au sens de la vue ${ }^{31}$. La constante ambigüité théorique entre une tactilité réelle, associée au toucher de la main, et une extension du caractère tactile aux fonctions de l'œil, n'a pas limité la portée de la relecture des principes qui constituent et déterminent le statut même de l'œuvre sculptée. Le débat dans lequel s'inscrit Hildebrand conduira ainsi, nonobstant les divers points de vue théoriques, à la révision radicale du rôle de la sculpture dans le panorama des arts contemporains.

Ce n'est pas un hasard si Rudolf Wittkower, dans son histoire de la sculpture ${ }^{32}$, articule la partie consacrée au XIX ${ }^{\mathrm{e}}$ siècle autour de deux seuls noms, celui de Rodin (Fig.5) et celui d'Hildebrand (Fig.6), le premier en tant qu'exemple du renouveau stylistique formel dans toute sa radicalité mais totalement éloigné d'un quelconque intérêt théorique, le second pour la force et la diffusion de son texte bien qu'il ne s'agisse pas d'un exemple de Belles lettres ${ }^{33}$.

Fig.5

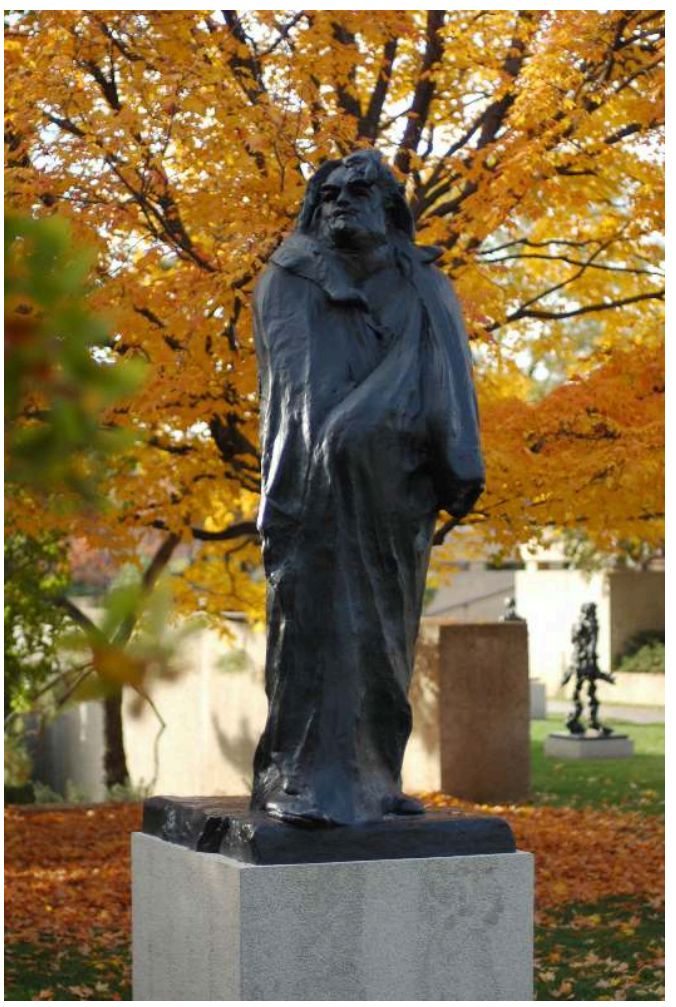

Auguste Rodin, Monument à Balzac, 1893-1938, Paris 
Fig.6

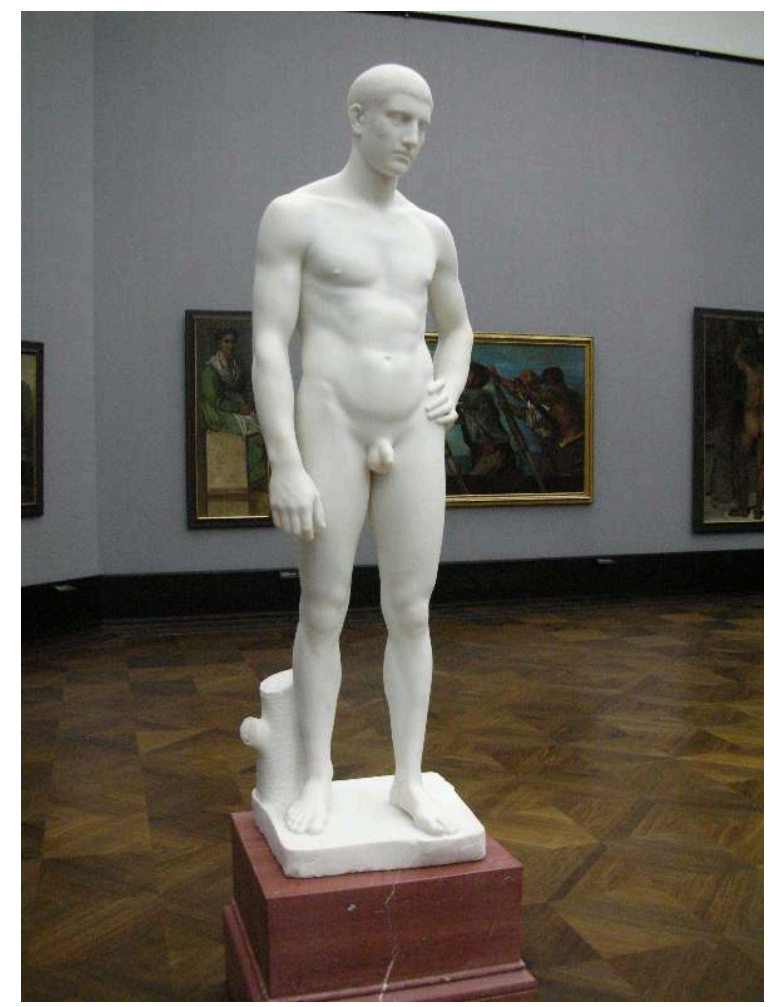

Adolf von Hildebrand, Stehenser Junger Mann, 1881-84, Berlin.

Le rapport entre ces deux auteurs est entièrement articulé autour de la conception d'ensemble d'une œuvre sculptée. Dans un article sur Rodin de 1918, Hildebrand lui reprochera l'accent mis sur le fragment, l'exaltation du détail, l'infinie multiplicité des plans. Il approuvera en revanche le caractère architectonique de l'image sculptée qui devait se conformer à la définition progressive par plans successifs de sa nature tridimensionnelle. C'était ainsi, selon Hildebrand, que procédait Michel-Ange lui-même à travers la technique de la taille directe de la pierre, que Hildebrand aurait voulu reprendre pour ses sculptures et qui constitue selon lui la technique du futur.

La leçon que Warburg reçoit du texte d'Hildebrand est centrée sur certains points fondamentaux qui apparaissent dans ses études des années suivantes. La notion de contrôle, liée à celle de "vision à distance“, est associée au caractère monumental de l'image.

41 C'est précisément lors d'une conférence en 1978, sur le thème de la Sculpture en extérieur, que Gombrich rappelle «la note énigmatique mais profonde d'Aby Warburg sur l'image : "Tu es vive mais tu ne me feras pas de mal", Du lebst und tust mir nichts. Je considère que cette étrange dualité est toujours présente in nuce quelle que soit la sculpture tridimensionnelle placée en extérieur si elle n'a pas encore subi - de nouveau une observation warburgiennene et benjaminienne - le processus d'esthétisation ${ }^{34}$.

Hildebrand reconnaît un pouvoir à l'image sculptée en extérieur : quand il identifie un rapport entre la figure plastique, caractérisée par sa valeur cubique, et un sentiment incontrôlable d'angoisse, il en vient à s'opposer au principe d'installation de statues dans des espaces publics. Pour Warburg, le thème du contrôle devait emprunter deux directions parallèles: l'une apte à conserver la valeur du sujet représenté dans la 
mémoire collective et culturelle, l'autre en mesure de maintenir constante et donc inaltérable cette valeur à travers la stabilité expressive des formes.

Les deux expressions « Monumentalisation » et «Prise de distance » sont associées par Warburg dans les notes de la planche 30 de l'Atlas (Fig.7), à propos des Batailles de Piero della Francesca à Arezzo.

Fig.7

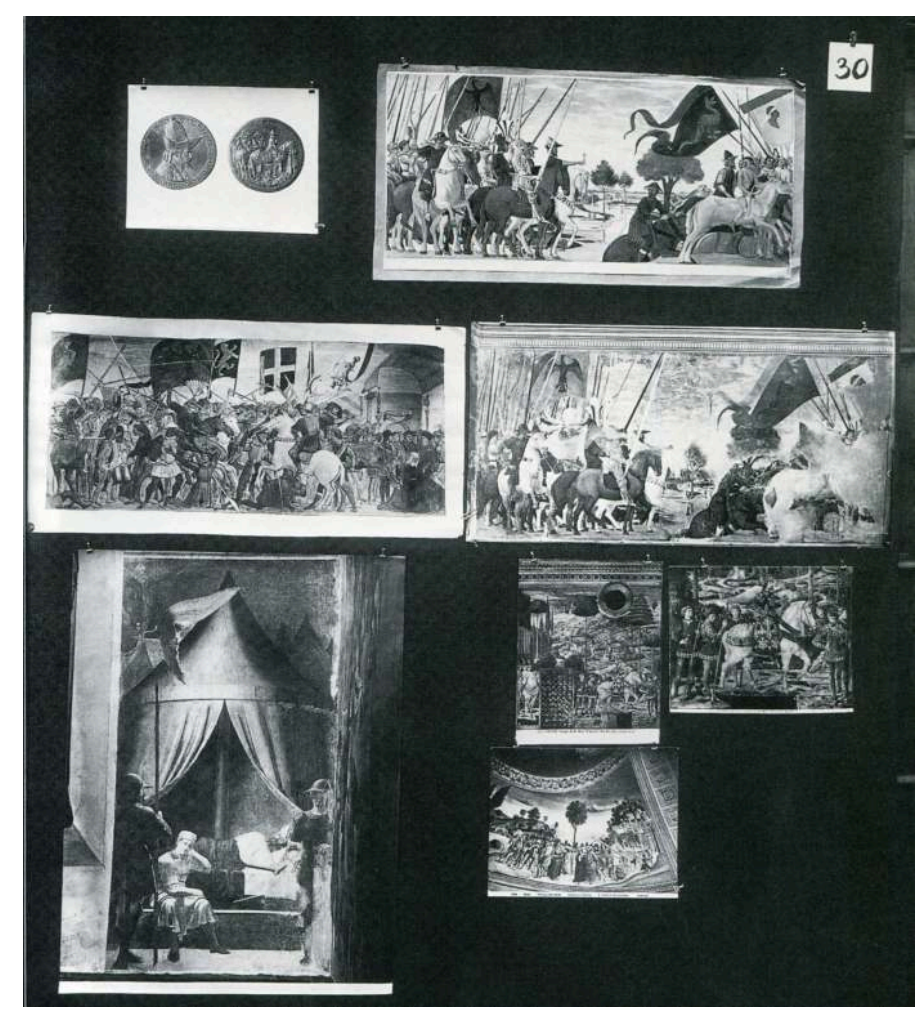

Aby Warburg, Mnemosyne, tav. 30

Il clarifie ces deux concepts, tant dans l'Introduction de l'Atlas (1927-1929) ${ }^{35}$, publiée pour la première fois en 1937, que dans l'essai de 1914 sur L'entrée du style idéal antiquisant dans la peinture du début de la Renaissance. Il existe dans la peinture de Piero "une antihéroïque de la figure isolée » afin de faire naître un style «dont la monumentalité héroïque transfigure le réalisme à des fins illustratives, sans pour autant l'éliminer $»^{36}$.

«Le recours à une forme de langue ancienne » auquel Warburg fait référence dans son analyse du monument, est à la base du processus d'adaptation de l'image caractérisée par un parcours de «transfiguration » du réel, et, en même temps, d'une tendance à la « monumentalisation » et à la " grandeur antihéroïque » du sujet. Ces deux aspects sont parties intégrantes de cette "prise de distance" autour de laquelle s'articule le contrôle de l'image. On retrouve une fois de plus ces concepts dans les notes de la planche 49 (Fig.8) dédiée à la grisaille interprétée par Warburg comme une métaphore et forme de contrôle du pathos dompté par l'image du vainqueur, et, dans un sens diamétralement opposé (" perte du "comme si" de la métaphore ») dans la planche 45 (Fig.9), où cette perte est associée aux "superlatifs dans le langage des signes », mais surtout à « l'exaltation de la conscience de soi » et au personnage/concept du « héros individuel qui s'éloigne de la grille typologique ». 
Fig.8

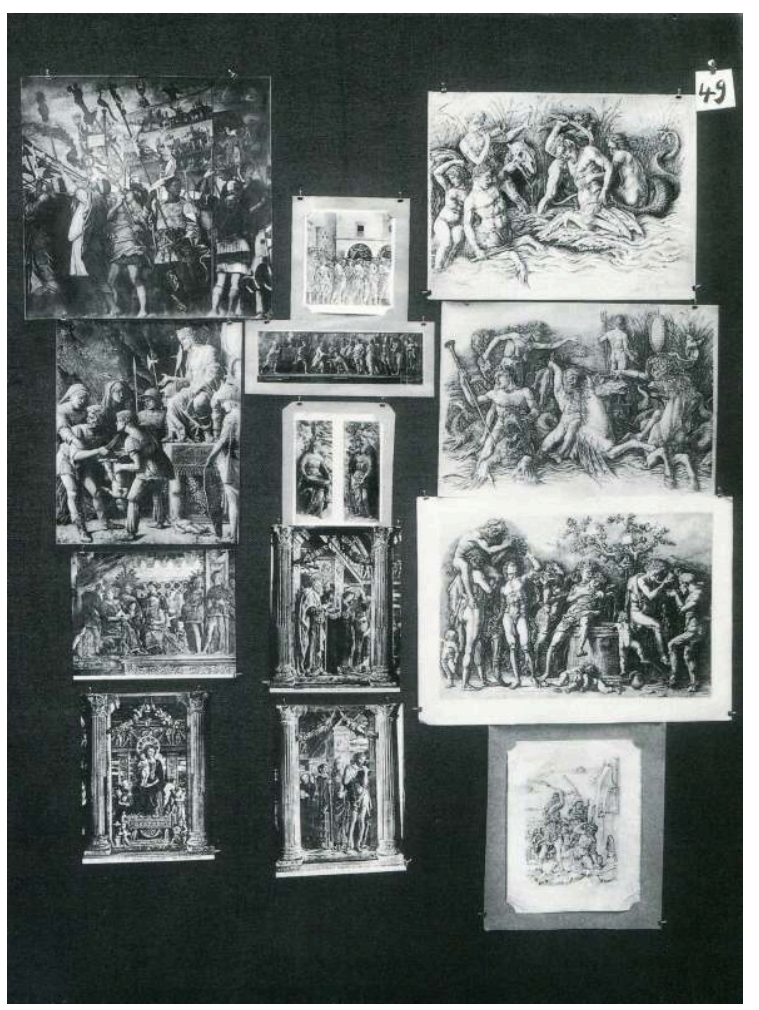

Aby Warburg, Mnemosyne, tav. 49.

Fig. 9

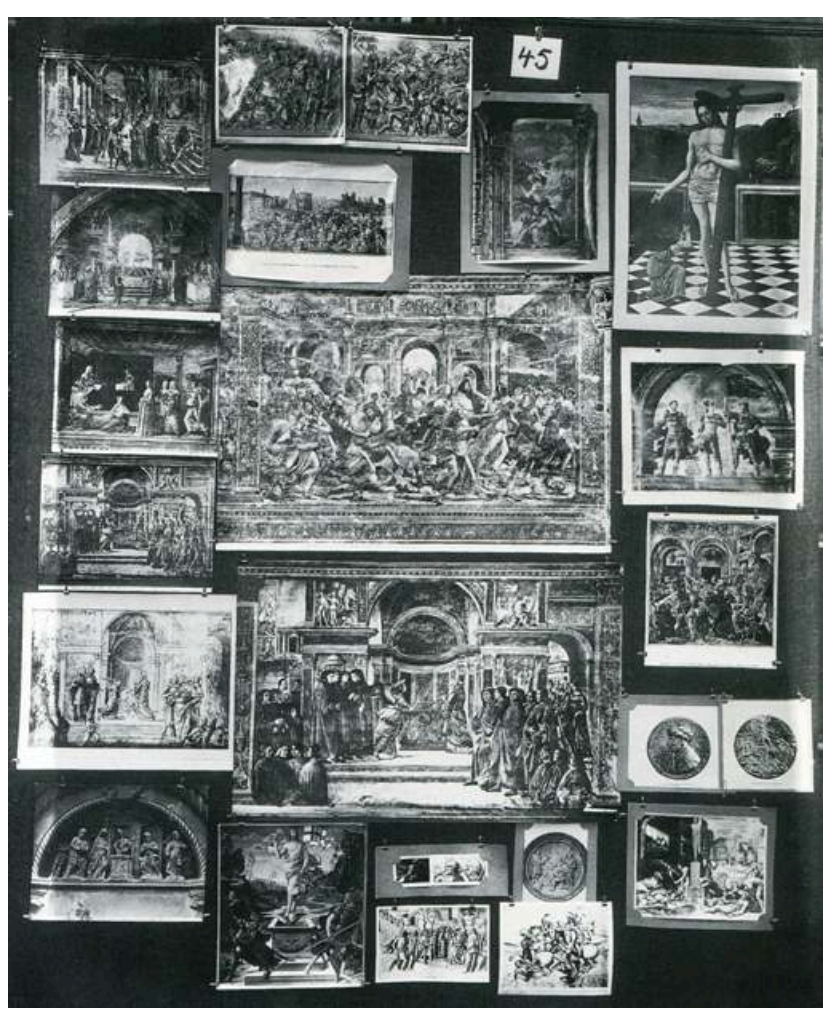

Aby Warburg, Mnemosyne, tav. 45. 
46 dire principe typologique, structurel de la composition - et métaphore, entre image formellement contrôlée et sa capacité à "être à la place de", et en déplaçant la notion de héros du champ de l'exaltation individuelle à celui d'une vision distanciée, et donc universellement acceptable de ce que le héros représente.

Dans le débat sur le monument à Bismarck, l'opposition "polaire" entre vision dionysiaque et apollinienne apparaît un caractère "primitif" de la forme essentielle associé à une valeur éthique qui trouve ses racines dans les travaux de Winckelmann. Le binôme composé, d'une part de l'essentiel formel, de l'autre de l'éthique de l'image, ouvre la voie à cette esthétique du signe qui joue un rôle fort complexe dans la pensée warburgienne, en s'imbriquant dans les recherches artistiques et philosophiques des débuts du XXe siècle.

Le processus d'adaptation de l'image conduit à la disparition de la "description" du geste typique, de l'attribut, de l'anecdote, mais pas de ce qui dans ce geste, cet attribut ou cette anecdote, appartient à la mémoire collective. Cette « langue ancienne est un expédient de l'artiste dans sa tentative de placer [...] son œuvre au niveau de la réalité. Si cette forme symbolique réussit toutefois, nonobstant son étroitesse à "vivre", la restriction devient un fluide de transmission très fort ". L'accès au signe, comme le pressent Warburg, n'est pas un éloignement du système de communication visuelle hautement stratifié comme un "mur palimpseste" - sur lequel les époques successives ne couvrent ni ne cachent jamais les précédentes - même si c'est un domaine particulier de lecture et réinterprétation, peut-être un point d'équilibre, dans le dialogue entre passé, mémoire et présent.

Ce n'est pas un hasard que la réflexion sur ces arguments parte d'un monument contemporain, commémoratif d'un personnage public perçu comme un héros. L'œuvre contemporaine devient un important et peut-être incontournable terrain de vérification des questions méthodologiques que Warburg s'employait à approfondir, en particulier au sujet de la définition ou plutôt de la notion de "symbole » qui apparaît dans l'une des deux dossiers de notes dédiées à l'art contemporain, incluant le Monument à Bismarck ${ }^{37}$.

Placé dans ce contexte, le symbole ne se limite pas à converger avec l'image contrôlée, « au-delà du temps », à travers le geste artistique qui coïncide avec une "prise de distance", mais trouve également un mode possible de communication dans le signe, pris dans son acception de métaphore, de “comme si”. C'est en ce sens que Warburg pense possible un Nachleben, un pouvoir de survivance de l'image formellement essentielle, en-dehors de son temps et en-dehors du souvenir individuel de celui qui a connu Bismarck. Le Nachleben, possible dans la mémoire de l'histoire est possible aussi par rapport à une œuvre contemporaine, à partir de l'appartenance critique et consciente au présent.

Images Re-vues, Hors-série 4 | 2013 


\section{NOTES}

1. A. Warburg, "Walpurgisnacht auf dem Stintfang ", in Hamburgischen Correspondenten, 29 janvier 1902. Le texte dactylographié est à Londres, Warburg Institute Archive [WIA], III.27.1.[1]. 2. Pour la documentation recueillie par Warburg voir WIA, IV.32.[1-4]. Toute la documentation relative au concours, y compris l'ensemble des projets présentés, est conservée aux Archives communales de Hambourg.

3. WIA, General Correspondence [GC], lettre d'E. Marcks à Aby Warburg du 4 septembre 1929, inv. 16342, dans laquelle il décline l'invitation du fait de nombreuses obligations.

4. J. Grand-Carteret, Bismarck en caricatures, avec 140 reproductions de caricatures allemandes, autrichiennes, françaises, italiennes, anglaises, suisses, américaines, Perrin et C. Éditeurs, Paris 1890. Avec ex-libris de Warburg.

5. M. A. Russell, Between tradition and modernity. Aby Warburg and the public purposes of art in Hamburg, 1896-1918. Berghahn Books, New York-Oxford 2007.

6. J. Schilling, "Distanz halten». Das Hamburger Bismarckdenkmal und die Monumentalität der Moderne, Wallstein Verlag, Göttingen 2006 ; id. (sous la direction de), Das Bismarckdenkmal in Hamburg 1906-2006, Beiträge zum Symposium “Distanz halten”. 100 Jahre Hamburger Bismarckdenkmal, Arbeitshefte zur Denkmalpflege in Hamburg, n. 24, Boyens Buchverlag, Hamburg 2008. Voir aussi: H.-E. Mitting e V. Plagemann, Denkmäler in 19.Jahrhundert. deutung und Kritik, Prestel-Verlag, München 1972, notamment V. Plagemann, Bismarck-Denkmäler, pp. 217-441; J. Traeger, Der Weg nach Walhalla. Denkmallandschaft und Bilddunsreise im 19. Jahrhuhdert, Bernhard Bosse Verlag, Regensburg 1991; U. Schlie, German Memorials. In Search of a Difficult Past. Nation and National Monuments in 19th and 20th Century German History, Goethe-Institute Inter Nationes, Marianne Exner, Bonn 2000.

7. C. Wedepohl, Walpurgisnacht auf dem Stintfang. Aby Warburg kunst-politisch, in J. Schilling (sous la direction de), Das Bismarckdenkmal in Hamburg 1906-2006, pp. 60-68.

8. E.H. Gombrich, «Standards of Truth: The Arrested Image and the Moving Eye ", dans W.J.T. Mitchell, The Language of Images, University of Chicago Press, 1980, pp. 181-218, réimprimé dans Critical Inquiry, Vol, 7, No. 2, winter 1980, pp. 237-273, et dans The Image and the Eye, Oxford 1982, pp. 244-277.

9. L. Marin, Politiques de la représentation, Éditions Kimé, Paris 2005, p. 72.

10. L. De Jaucourt, Monument, en Encyclopédie, ou Dictionnaire raisonné des sciences, des arts et des métiers par une société de gens de lettres, sous la direction de D. Diderot et J.B. D’Alembert, Imprimerie des éditeurs, Livourne 1773, vol. X, tome II, pp. 636-637.

11. V. Plagemann, Bismarck-Denkmäler, in H.-E. Mitting et V. Plagemann, Denkmäler in 19. Jahrhundert, p. 235.

12. A. Warburg, L'art du portrait et la bourgeoisie florentine, in id., Essais florentins, Klincksieck, Paris 1990, pp. 101-136

13. Également dans une lettre à von Bode, directeur de la Gemäldegalerie de Berlin, adressée alors qu'il concevait son écrit. WIA, GC, lettre d'Aby Warburg à Wilhelm von Bode, du 10 décembre 1902, inv. 35031.

14. É. Zola, Ecrits sur l'art, Gallimard, Paris 1991, p. 244 : «Si un art souffre du milieu moderne, c'est à coup sûr la sculpture ».

15. J. K. Huysmans, L'art moderne ; Certains, UGE, Paris 1975, p. 89 : « De deux choses l'une, ou la sculpture peut s'acclimater avec la vie moderne, ou elle ne le peut pas ».

16. C. Baudelaire, Salon 1846, chap. XVI. Pourquoi la sculpture est ennuyeuse, in Id., Critique d'art, sous la direction de C. Pichois, Gallimard, Paris 1992, pp. 147-149; mais voir aussi salon 1859, chap. IX. Sculpture, ivi, pp. 329-340. 
17. Pour le débat en général, voir J. Lichtenstein, Pourquoi la sculpture ne pouvait pas être moderne?, in G. Roque, Majeur ou mineur. Les hiérarchies en art, Nîmes 2000; L. Russo (sous la direction de), Estetica della scultura, Aesthetica Edizioni, Palermo 2003.

18. Un ouvrage de H. Belting, Die Deutschen und ihre Kunst. Ein schwieriges Erbe, C. H. Beck, München 1992, reprend de façon très documentée ce double aspect (trad. anglaise, The Germans and their art. A troublesome relationship, Yale University Press, Yale 1998 ; trad. it., I Tedeschi e la loro arte. Un'eredità difficile, Il Castoro, Milan 2005).

19. Un terme, généralement réservé au domaine médical, utilisé en 1893 par Max Nordau dans son ouvrage intitulé Dégénération, où il fait une distinction entre artistes sains et artistes malades : M. Nordau, Entrartung, Verlag Carl Duncker, Berlin 1893, 2 volumes.

20. A. Warburg, Dürer et l'Antiquité italienne, in Essais florentins, sous la direction de E. Pinto, Klincksieck, Paris 1990, pp. 159-166.

21. Ib. pp. 163-164.

22. G. Bing, Introduzione, in A. Warburg, La rinascita del paganesimo antico, La Nuova Italia, Firenze 1966, pp. VII-XXXI, p. XXVII.

23. J. von Schlosser, Vom Modernen Denkmalkuktus, in «Vorträge der Bibliothek Warburg», VI, 1926-27, extrait, pp. 1-21.

24. J. Von Schlosser, Histoire du portrait en cire, postface de Th. Medicus, Macula, Paris, 1997. La traduction italienne de ce livre (Storia del ritratto in cera, a cura di P. Conte, Quodlibet, Macerata, 2011) est accompagnée par l'essai de G. Didi-Huberman, Viscosità e sopravvivenze. La storia dell'arte alla prova del materiale, pp. 7-30.

25. Cfr. C. Wedepohl, Walpurgisnacht auf dem Stintfang, p. 61, aussi pour les citations précédentes.

26. W. Benjamin, L'ceuvre d'art à l'époque de sa reproductibilité technique: Version de 1939, Gallimard, Paris 2008, chap. 2, par. 4.

27. A. Assmann, Erinnerungsräume. Formen und Wandlungen des Kulturellen Gedächtnisses, C.H. Beck, München 1999.

28. Cet essai ne figure pas dans l'édition française du recueil de Warburg, Essais florentins.

29. W. Fagus, « Der Roland von Hamburg », in Deutsche Welt, 10 juin 1906, pp. 577-578.

30. A. von Hildebrand, Le problème de la forme dans les arts plastiques, Éditions L'Harmattan, Paris 2003.

31. Cfr. P. D’Angelo, « Dal Settecento a oggi », in L. Russo (sous la direction de), Estetica della Scultura, pp. 91-125.

32. R. Wittkower, Sculpture : Processes and Principles, Lane, London 1977, trad. fr, Qu'est-ce que la sculpture ?: Principes et procédures de l'Antiquité au XXe siècle, Macula, Pair, 2000.

33. En 1914 il était à sa IXème édition.

34. E.H. Gombrich, Sculpture for Outdoors, in id., The Uses of Images : Studies in the Social Function of Art and Visual Communication, Phaidon Press Limited, London 1999, pp.136-153 (traduction française de l'auteur).

35. La première version du texte a été rassemblée par le dit Geburtstagsatlas, sous la direction de G. Bing, E. H. Gombrich, F. Saxl, 1937 [WIA III.109]; Einleitung zum Mnemosyne-Atlas, in Die Beredsamkeit des Leibes. Zur Körpersprache in der Kunst, sous la direction d'I. Barta-Fliedl, C. Geissmar-Brandi, Residenz Verlag, Salzbourg-Vienne 1992, pp. 171-173; trad. it. de G. Sampaolo in Mnemosyne. L'Atlante della Memoria di Aby Warburg, travaux sous la direction d'I. Spinelli, R. Venuti, éd. Artemide, Rome 1998, pp. 23-26; version du texte basée sur P. van Huisstede, De Mnemosyne Beeldatlas van Aby Warburg : een laboratorium voor beeldgeschiedenis, Proefschrift ter verkrijging van de graad van Doctor aan de Rijsuniversiteit te Leiden, 3 décembre 1992 (GS II.1, pp. 3-6 ; AWO II.1, pp. 3-5); trad. it. Mnemosyne. L'Atlante delle immagini, sous la direction de M. Warnke, édition italienne sous la direction de M. Ghelardi, éd. Aragno, Turin 2002; éd. critique in Aby M. Warburg. Mnemosyne Materialen, sous la direction de W. Rappl, G. Swodoba, W. Pichler, M. Koos,MünichHambourg 2006. 
36. A. Warburg, L'entrée du style idéal antiquisant dans la peinture du début de la Renaissance, in Essais florentins, pp. 221-243, citation p. 228.

37. WIA, III.27.2.2, folios 1, 3, 4, 4a.

INDEX

Mots-clés : débat, sculpture, Allemagne XIX-XX siècles, identité, mémoire, monument

\section{AUTEUR}

\section{MICOL FORTI}

Directrice de la collection d'art contemporain des musées du Vatican, elle a enseigné à l'université de Rome «La Sapienza » et a édité avec Claudia Cieri Via, Aby Warburg e la cultura italiana. Fra sopravvivenze e prospettive di ricerca. 